\title{
Eigenvibrations of a simply supported beam with elastically attached load
}

\author{
Anton A. Samsonov ${ }^{1}$, Sergey I. Solov'ev ${ }^{1, *}$, and Pavel S. Solov'ev ${ }^{1}$ \\ ${ }^{1}$ Kazan Federal University, 18 Kremlevskaya Street, Kazan 420008, Russian Federation
}

\begin{abstract}
The nonlinear differential eigenvalue problem describing eigenvibrations of a simply supported beam with elastically attached load is investigated. The existence of an increasing sequence of positive simple eigenvalues with limit point at infinity is established. To the sequence of eigenvalues, there corresponds a system of normalized eigenfunctions. To illustrate the obtained theoretical results, the initial problem is approximated by the finite difference method on a uniform grid. The accuracy of approximate solutions is studied. Investigations of the present paper can be generalized for the cases of more complicated and important problems on eigenvibrations of plates and shells with elastically attached loads.
\end{abstract}

\section{Introduction}

Let us formulate the nonlinear differential eigenvalue problem governing eigenvibrations of the beam-spring-load system. Assume that the beam axis occupies in the equilibrium horizontal position the segment $[0, l]$ on the $O x$ axis. Denote by $\rho(x)$ the volume mass density, $E(x)$ the elasticity Young modulus, $S(x)$ the square of transversal cut of the beam, $J(x)$ the inertia moment of the cut with respect to its horizontal axis at the point $x \in[0, l]$. Suppose that the ends $x=0$ and $x=l$ of the beam are simply supported, at a point $x^{(0)} \in(0, l)$ of the beam a load of mass $M$ is joined by elastic spring with the stiffness coefficient $K$. Then the vertical deflection $w(x, t)$ of the beam at a point $x$ at time $t$ and the vertical deflection $\eta(t)$ of the load of mass $M$ at time $t$ satisfy the following equations

$$
\begin{gathered}
\frac{\partial^{2}}{\partial x^{2}}\left(p(x) \frac{\partial^{2}}{\partial x^{2}} w(x, t)\right)+r(x) \frac{\partial^{2}}{\partial t^{2}} w(x, t)=0, x \in\left(0, x^{(0)}\right) \cup\left(x^{(0)}, l\right), t>0, \\
w(0, t)=\frac{\partial^{2}}{\partial x^{2}} w(0, t)=0, w(l, t)=\frac{\partial^{2}}{\partial x^{2}} w(l, t)=0, t>0
\end{gathered}
$$

\footnotetext{
*Corresponding author: sergei.solovyev@kpfu.ru
} 


$$
\begin{gathered}
{[w(x, t)]_{x=x^{(0)}}=\left[\frac{\partial}{\partial x} w(x, t)\right]_{x=x^{(0)}}=\left[\frac{\partial^{2}}{\partial x^{2}} w(x, t)\right]_{x=x^{(0)}}=0, t>0} \\
{\left[\frac{\partial}{\partial x}\left(p(x) \frac{\partial^{2}}{\partial x^{2}} w(x, t)\right)\right]_{x=x^{(0)}}=K\left(\eta(t)-w\left(x^{(0)}, t\right)\right), t>0} \\
M \eta^{\prime \prime}(t)+K\left(\eta(t)-w\left(x^{(0)}, t\right)\right)=0, t>0
\end{gathered}
$$

where $[w(x, t)]_{x=x^{(0)}}=w\left(x^{(0)}+0, t\right)-w\left(x^{(0)}-0, t\right), \quad p(x)=E(x) J(x), \quad r(x)=\rho(x) S(x)$, $x \in[0, l], t>0$.

The eigenvibrations of the mechanical beam-spring-load system are characterized by the functions $w(x, t)$ and $\eta(t)$ of the form

$$
w(x, t)=u(x) \sin (\omega t), \eta(t)=z \sin (\omega t), x \in[0, l], t>0,
$$

where $z$ and $\omega$ are constants. The number $\omega$ determines the eigenvibration frequency of the beam-spring-load system, the function $u(x), x \in[0, l]$, determines the eigenvibration amplitude at each point $x \in[0, l]$ of the beam, the number $z$ is the eigenvibration amplitude of the load, and the function $\sin (\omega t), t>0$, describes the motion in time of points of the mechanical beam-spring-load system under the eigenvibration.

From (5) and (6), we obtain the relation

$$
z(\sigma-\lambda)-\sigma u\left(x^{(0)}\right)=0
$$

where $\sigma=K / M, \lambda=\omega^{2}$. If $\lambda \neq \sigma$, then from relation (7) we obtain $z=\sigma u\left(x^{(0)}\right) /(\sigma-\lambda)$, and equations (1)-(4) lead to the following nonlinear eigenvalue problem: find numbers $\lambda$ and nonzero functions $u(x), x \in[0, l]$, such that

$$
\begin{gathered}
\left(p(x) u^{\prime \prime}(x)\right)^{\prime \prime}=\lambda r(x) u(x), x \in(0, l), \\
u(0)=u^{\prime \prime}(0)=0, u(l)=u^{\prime \prime}(l)=0, \\
{[u(x)]_{x=x^{(0)}}=\left[u^{\prime}(x)\right]_{x=x^{(0)}}=\left[u^{\prime \prime}(x)\right]_{x=x^{(0)}}=0,} \\
{\left[\left(p(x) u^{\prime \prime}(x)\right)^{\prime}\right]_{x=x^{(0)}}=\frac{\lambda}{\sigma-\lambda} K u\left(x^{(0)}\right) .}
\end{gathered}
$$

If $\lambda=\sigma$, then from (7) we get the relation $u\left(x^{(0)}\right)=0$, and equations (1)-(4) lead to the following eigenvalue problem for $\lambda^{(0)}=\sigma$ : find nonzero functions $u(x), x \in[0, l]$, such that

$$
\left(p(x) u^{\prime \prime}(x)\right)^{\prime \prime}=\lambda^{(0)} r(x) u(x), x \in(0, l),
$$




$$
\begin{gathered}
u(0)=u^{\prime \prime}(0)=0, u\left(x^{(0)}\right)=0, u(l)=u^{\prime \prime}(l)=0, \\
{[u(x)]_{x=x^{(0)}}=\left[u^{\prime}(x)\right]_{x=x^{(0)}}=\left[u^{\prime \prime}(x)\right]_{x=x^{(0)}}=0,}
\end{gathered}
$$

In the present paper, the existence of an increasing sequence of positive simple eigenvalues with limit point at infinity is proved for the nonlinear eigenvalue problem (8)(11). To the sequence of eigenvalues, there corresponds a system of normalized eigenfunctions. Our study of the limit properties of auxiliary parametric spectral problems allows considering problem (12)-(14) as an extension of problem (8)-(11) to $\lambda=\sigma$. To illustrate the obtained theoretical results, the initial problem is approximated by the finite difference method on a uniform grid. The accuracy of approximate solutions is studied.

Nonlinear eigenvalue problems have numerous applications in science and engineering [1-6]. Computational methods for solving nonlinear matrix eigenvalue problems were studied in [7-15]. The error of the finite difference method for solving differential eigenvalue problems with nonlinear dependence on the spectral parameter was investigated in $[1,17]$. For nonlinear differential spectral problems, the finite element method was studied in [18-20] based on the use general results in the linear case [21-23]. Approximate methods for solving applied nonlinear boundary value problems and variational inequalities have been investigated in the papers [24-30].

\section{Variational statement of the problem}

Let $\mathbb{R}$ be the real line, and let $\Omega=(0, l)$ and $\bar{\Omega}=[0, l]$. As usual, by $L_{2}(\Omega)$ and $W_{2}^{2}(\Omega)$ we denote the real Lebesgue and Sobolev spaces equipped with the norms $|\cdot|_{0}$ and $\|\cdot\|_{2}$, respectively, where

$$
\begin{gathered}
|v|_{0}=\left(\int_{0}^{l}(v(x))^{2} \mathrm{~d} x\right)^{1 / 2}, \\
\|v\|_{2}=\left(|v|_{0}^{2}+|v|_{1}^{2}+|v|_{2}^{2}\right)^{1 / 2}, \quad|v|_{1}=\left|v^{\prime}\right|_{0}, \quad|v|_{2}=\left|v^{\prime \prime}\right|_{0} .
\end{gathered}
$$

Set $H=L_{2}(\Omega)$ and $V=\left\{v: v \in W_{2}^{2}(\Omega), v(0)=v(l)=0\right\}$. Define sufficiently smooth functions $p(x), r(x), x \in \bar{\Omega}$, for which there exist positive constants $p_{i}, \quad r_{i}, \quad i=1,2$, such that $p_{1} \leq p(x) \leq p_{2}, \quad r_{1} \leq r(x) \leq r_{2}$. Introduce positive numbers $M, K, x^{(0)} \in \Omega$, and bilinear forms $a: V \times V \rightarrow \mathbb{R}, b: H \times H \rightarrow \mathbb{R}, c: V \times V \rightarrow \mathbb{R}$, by the formulas

$$
a(u, v)=\int_{0}^{l} p(x) u^{\prime \prime}(x) v^{\prime \prime}(x) \mathrm{d} x, b(u, v)=\int_{0}^{l} r(x) u(x) v(x) \mathrm{d} x, c(u, v)=K u\left(x^{(0)}\right) v\left(x^{(0)}\right) .
$$

Denote $\sigma=K / M, \Lambda_{1}=(0, \sigma), \Lambda_{2}=(\sigma, \infty), \Lambda_{0}=\Lambda_{1} \cup \Lambda_{2}, \Lambda=(0, \infty)$. Put

$$
\varsigma(\mu)=\frac{1}{\sigma-\mu}, \mu \in \Lambda_{1}, \xi(\mu)=\frac{\mu}{\mu-\sigma}, \mu \in \Lambda_{2} .
$$

The differential nonlinear eigenvalue problem (8)-(11) is equivalent to the following variational nonlinear eigenvalue problem: find $\lambda \in \Lambda_{0}, u \in V \backslash\{0\}$, such that 


$$
a(u, v)+\xi(\lambda) c(u, v)=\lambda b(u, v) \forall v \in V .
$$

The variational problem (19) is equivalent to the following nonlinear eigenvalue problem: find $\lambda \in \Lambda_{0}, u \in V \backslash\{0\}$, such that

$$
a(u, v)=\lambda(b(u, v)+\varsigma(\lambda) c(u, v)) \forall v \in V .
$$

\section{Parameter eigenvalue problems}

Introduce the following parameter dependent linear eigenvalue problems for fixed parameter $\mu \in \Lambda$.

Find $\varphi(\mu) \in \mathbb{R}, u \in V \backslash\{0\}$, such that

$$
a(u, v)=\varphi(\mu)(b(u, v)+\varsigma(\mu) c(u, v)) \forall v \in V
$$

for fixed parameter $\mu \in \Lambda_{1}$.

Find $\psi(\mu) \in \mathbb{R}, u \in V \backslash\{0\}$, such that

$$
a(u, v)+\xi(\mu) c(u, v)=\psi(\mu) b(u, v) \forall v \in V
$$

for fixed parameter $\mu \in \Lambda_{2}$.

For fixed parameter $\mu \in \Lambda_{1}$, problem (21) has an increasing sequence of positive simple eigenvalues with limit point at infinity $\varphi_{k}(\mu), k=1,2, \ldots$ For fixed parameter $\mu \in \Lambda_{2}$, problem (22) has an increasing sequence of positive simple eigenvalues with limit point at infinity $\psi_{k}(\mu), k=1,2, \ldots$ The functions $\varphi_{k}(\mu), \mu \in \Lambda_{1}, k=1,2, \ldots$, and $\psi_{k}(\mu), \quad \mu \in \Lambda_{2}, \quad k=1,2, \ldots$, are continuous and non-increasing.

Define the subspace $V_{0}=\left\{v: v \in V, v\left(x^{(0)}\right)=0\right\}$ of the space $V$. Introduce the following linear eigenvalue problem.

Find $\lambda^{(0)} \in \mathbb{R}, u \in V_{0} \backslash\{0\}$, such that

$$
a(u, v)=\lambda^{(0)} b(u, v) \forall v \in V_{0} .
$$

Problem (23) has an increasing sequence of positive simple eigenvalues with limit point at infinity $\lambda_{k}^{(0)}, k=1,2, \ldots$ Put $\lambda_{0}^{(0)}=0$.

Theorem 1. The following limit properties hold: $\varphi_{k}(\mu) \rightarrow \lambda_{k-1}^{(0)}$ as $\mu \rightarrow \sigma-0$, $\psi_{k}(\mu) \rightarrow \lambda_{k}^{(0)}$ as $\mu \rightarrow \sigma+0$.

The proof of this theorem generalizes results from [5].

\section{Existence of eigensolutions}

Set $\psi_{0}(\mu)=0$ for $\mu \in[\sigma, \infty), \quad \psi_{k}(\sigma)=\lambda_{k-1}^{(0)}, \quad k=1,2, \ldots$, and define $\gamma_{k}(\mu), \quad \mu \in \Lambda$, $k=1,2, \ldots, \quad$ by the formulas $\gamma_{k}(\mu)=\varphi_{k}(\mu), \quad \mu \in(0, \sigma), \quad$ and $\quad \gamma_{k}(\mu)=\psi_{k-1}(\mu)$, $\mu \in[\sigma, \infty)$, for $k=1,2, \ldots$

Theorem 2. The functions $\gamma_{k}(\mu), \mu \in \Lambda, k=1,2, \ldots$, are continuous and nonincreasing. 
The proof of this theorem follows from properties of the functions $\varphi_{k}(\mu), \mu \in \Lambda_{1}$, $k=1,2, \ldots$, and $\psi_{k}(\mu), \mu \in \Lambda_{2}, k=1,2, \ldots$

Theorem 3. Problem (19) has an increasing sequence of positive simple eigenvalues with limit point at infinity $\lambda_{k}, k=1,2, \ldots$ Each eigenvalue $\lambda_{k}, k \geq 1$, is a unique root of the equation $\mu-\gamma_{k}(\mu)=0, \mu \in \Lambda, k \geq 1$. To the sequence of eigenvalues $\lambda_{k}, k=1,2, \ldots$, there corresponds a system of normalized eigenfunctions $u_{k}, b\left(u_{k}, u_{k}\right)=1, \quad k=1,2, \ldots$

The proof of this theorem generalizes results from [5].

\section{Numerical experiments}

Introduce the partition of the segment $[0, l]$ by equidistant points $x_{i}=i h, i=0,1, \ldots, N$, $h=l / N$. Put $x_{-1}=-h, x_{N+1}=l+h$. Assume that $x^{(0)}=x_{m}$ for some $m, 0<m<N$. Denote $\quad p_{i}=p\left(x_{i}\right), \quad r_{i}=r\left(x_{i}\right), \quad \delta_{i i}=1, \quad \delta_{i j}=0, \quad i \neq j, \quad y_{x, i}=\left(y_{i+1}-y_{i}\right) / h$, $y_{\bar{x}, i}=\left(y_{i}-y_{i-1}\right) / h, i, j=0,1, \ldots, N$. Approximate problem (8)-(11) by the following finite difference scheme

$$
\begin{gathered}
\left(p y_{\bar{x} x}\right)_{\bar{x} x, i}=\lambda^{h}\left(r_{i}+\frac{\delta_{m i}}{h} \frac{K}{\sigma-\lambda^{h}}\right) y_{i}, i=1,2, \ldots, N-1, \\
y_{0}=y_{\bar{x} x, 0}=0, y_{N}=y_{\bar{x} x, N}=0 .
\end{gathered}
$$

Problem (24), (25) has eigenvalues $\lambda_{1}^{h} \leq \lambda_{2}^{h} \leq \ldots \leq \lambda_{N}^{h}$. The following error estimate is valid $\left|\lambda_{k}^{h}-\lambda_{k}\right| \leq c h^{2}$, where $c$ is a positive constant.

To illustrate theoretical results of Theorems 1 and 2, we have solved the eigenvalue problem (19) for $p(x)=1, \quad r(x)=1, \quad x \in[0, l], \quad l=1, M=0.1, \quad K=200, \quad \sigma=2000$, applying the finite difference scheme (24), (25) for $x^{(0)}=5 / 26, N=26$. Fig. 1 shows the graphs of the functions $\gamma_{i}(\mu), i=1,2,3$, the eigenvalues $\lambda_{i}, i=1,2,3$, of the nonlinear eigenvalue problem (19), the eigenvalues $\lambda_{i}^{(0)}, i=1,2$, of the limit linear eigenvalue problem (23). We see that the experimental results are consistent with the theoretical results
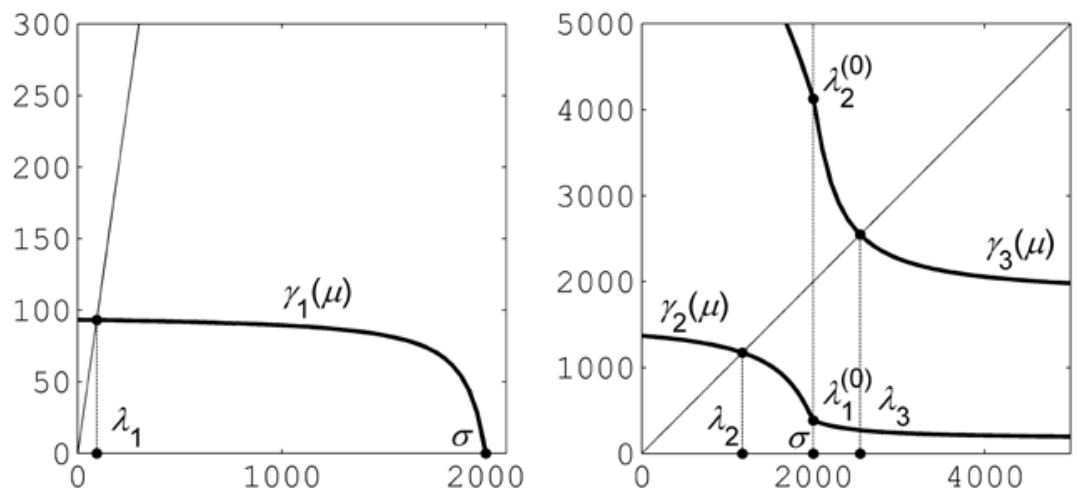

Fig. 1. Eigenvalues of the nonlinear eigenvalue beam problem. 
of Theorems 1, 2, and 3; namely, $\gamma_{1}(\mu) \rightarrow 0$ as $\mu \rightarrow \sigma, \quad \gamma_{i+1}(\mu) \rightarrow \lambda_{i}^{(0)}$ as $\mu \rightarrow \sigma, i=1,2$. Investigations of the present paper can be generalized for the problems on eigenvibrations of plates and shells with elastically attached loads.

This work was supported by Russian Science Foundation, project no. 16-11-10299.

\section{References}

1. A.V. Goolin, S.V. Kartyshov, Surv. Math. Ind. 3, 29-48 (1993)

2. T. Betcke, N.J. Higham, V. Mehrmann, C. Schröder, F. Tisseur, ACM Trans. Math. Software 39, 7 (2013)

3. V.A. Kozlov, V.G. Maz'ya, J. Rossmann, Spectral Problems Associated with Corner Singularities of Solutions to Elliptic Equations (AMS, Providence, 2001)

4. E.S. Karchevskii, S.I. Solov'ev, Differ. Equations 36, 631-634 (2000)

5. S.I. Solov'ev, Lobachevskii J. Math. 37, 597-609 (2016)

6. S.I. Solov'ev, Izv. Vyssh. Uchebn. Zaved. Mat. 10, 71-74 (1985)

7. R.Z. Dautov, A.D. Lyashko, S.I. Solov'ev, Russ. J. Numer. Anal. Math. Modelling 9, 417-427 (1994)

8. A. Ruhe, SIAM J. Numer. Anal. 10, 674-689 (1973)

9. V. Mehrmann, H. Voss, GAMM-Mit. 27, 1029-1051 (2004)

10. F. Tisseur, K. Meerbergen, SIAM Rev. 43, 235-286 (2001)

11. D. Kressner, Numer. Math. 114, 355-372 (2009)

12. X. Huang, Z. Bai, Y. Su, J. Comput. Math. 28, 218-234 (2010)

13. H. Schwetlick, K. Schreiber, Linear Algebra Appl. 436, 3991-4016 (2012)

14. W.-J. Beyn, Linear Algebra Appl. 436, 3839-3863 (2012)

15. A. Leblanc, A. Lavie, Eng. Anal. Bound. Elem. 37, 162-166 (2013)

16. X. Qian, L. Wang, Y. Song, J. Comput. Appl. Math. 290, 268-277 (2015)

17. A.V. Kregzhde, Differ. Uravn. 17, 1280-1284 (1981)

18. S.I. Solov'ev, Differ. Equations 50, 947-954 (2014)

19. S.I. Solov'ev, Differ. Equations 51, 934-947 (2015)

20. R.Z. Dautov, A.D. Lyashko, S.I. Solov'ev, Differ. Equations 27, 799-806 (1991)

21. S.I. Solov'ev, Differ. Equations 30, 1138-1146 (1994)

22. S.I. Solov'ev, Differ. Equations 38, $752-753$ (2002)

23. S.I. Solov'ev, Appl. Numer. Math. 93, 206-214 (2015)

24. I.B. Badriev, G.Z. Garipova, M.V. Makarov, V.N. Paymushin, Res. J. Appl. Sciences 10, 428-435 (2015)

25. I.B. Badriev, L.A. Nechaeva, PNRPU Mech. Bull. 3, 37-65 (2013)

26. I.B. Badriev, M.V. Makarov, V.N. Paimushin, Proc. Engin. 150, 1050-1055 (2016)

27. I.B. Badriev, G.Z. Garipova, M.V. Makarov, V.N. Paimushin, R. F. Khabibullin, Lobachevskii J. Math. 36, 474-481 (2015)

28. I.B. Badriev, M.V. Makarov, V.N. Paimushin, Russ. Math. 59, 57-60 (2015)

29. I.B. Badriev, M.V. Makarov, V.N. Paimushin, Proc. Engin. 150, 1056-1062 (2016)

30. I.B. Badriev, V.V. Banderov, V.L. Gnedenkova, N.V. Kalacheva, A.I. Korablev, R.R. Tagirov, Appl. Math. Sciences 9, 5697-5705 (2015) 